\title{
Commentary on: "Lumbar Intervertebral Discal Cyst: A Rare Cause of Low Back Pain and Radiculopathy. Case Report and Review of the Current Evidences on Diagnosis and Management"
}

\author{
Marc D. Moisi ${ }^{1}$ Jeni Page ${ }^{1}$ Rod J. Oskouian, Jr. ${ }^{1}$ \\ ${ }^{1}$ Department of Neurosurgery, Swedish Neuroscience Institute, \\ Seattle, Washington, United States \\ Evid Based Spine Care J 2014;5:149-150.
}

An intradiscal cyst is one of the least common etiologies of lumbar radicular pain. Although it may appear that the pathophysiology is self-explanatory, the formation and natural history of the condition continues to elude surgeons because of its infrequency. Thus, literature and research regarding the topic is limited, making the development of an accurate understanding regarding the treatment of the discs quite challenging.

Certo et al illustrate a case of an intervertebral discal cyst that presented with lumbar pain and radiculopathy. Their report included an in-depth review of the literature with a total of 105 cases regarding management of these rare cysts.

Prior to considering surgical intervention, understanding of the formation and composition of the cysts is crucial to determining the best treatment modality. The authors discuss several instances in the literature that have attempted to define the consistency, as well the characteristics, of such cysts. Chiba et al have given a very thorough descriptive evaluation of such cysts, including location, symptomatology (unilateral nerve root), fluid content, and lack of disc material within the cystic lesion. However, in 2010, Kobayashi et $\mathrm{al}^{1}$ argued that in their two cases the histological features of the cyst were similar to the absorption process of a disc herniation, and the presence of residual herniated tissues was confirmed in the cyst wall. Thus, the actual pathological features regarding these lesions continue to remain elusive.

Despite questions regarding the formation and composition of the cyst, the diagnosis can be made and confirmed with magnetic resonance imaging (MRI) with and without contrast enhancement. On MRI, the cyst wall is contrast-enhancing and is connected to the disc space. We agree with the authors that MRI is the essential tool in the diagnosis of the discal cyst, but we do not find much added value to doing discography

received

July 2, 2014

accepted

July 2, 2014

Address for correspondence Marc D. Moisi, MD, Department of Neurosurgery, Swedish Neuroscience Institute, Seattle, WA, United States (e-mail: Marc.Moisi@swedish.org).

followed by a computed tomography (CT) scan as it would not significantly alter the management of the patient.

Ultimately, the most important part to learn from this review is how to manage such a patient if one presents himself to your office. In our opinion, the symptoms should determine the treatment. If the patient presents with tolerable pain without neurological deficits, conservative medical therapies and management should be the first line in management. Aydin et $\mathrm{al}^{2}$ discuss medical therapies, and although the failure rate was $62.5 \%$ with the ultimate result of surgery, there is value to conservatively managing a patient given the invasiveness of the operation. If an invasive strategy is necessary, an aspiration of the cyst versus a surgical technique should be weighed and considered. CT-guided aspirations of cysts, as detailed by Kang et al, ${ }^{3}$ demonstrated an $89 \%$ success rate (one out of the eight patients recurred). However, the location of the cyst will determine the feasibility of this treatment modality. The best success overall appears to be an open microsurgical technique with drainage and excision of the cyst as the authors illustrated in their case. We did disagree that a discectomy should be performed as removing the disc does not add any value to the decompressive element or to the possibility of recurrence. Instead, it potentiates the probability of a future disc rupture and could possibly increase the rate of disc degeneration. Therefore, we would recommend a decompression of the neural elements followed by drainage and removal of the cyst.

In conclusion, Certo et al have done a thorough job of compiling all the evidence and cases regarding discal cysts of the lumbar region. Although the spine surgical community still needs to come to a consensus regarding the pathophysiology and composition of such lesions, this article nicely demonstrates that there is an important role for intervention

(c) 2014 Georg Thieme Verlag KG Stuttgart · New York
DOI http://dx.doi.org/ 10.1055/s-0034-1387803. ISSN 1663-7976. 
with these patients. Nevertheless, each patient should be individually assessed based on their symptoms as to which intervention would be most beneficial.

\section{References}

1 Kobayashi S, Takeno K, Uchida K, et al. Pathogenesis of the discal cysts communicating with an adjacent herniated disc. Histological and ultrastructual studies of two cases. Joint Bone Spine 2010; 77(2):184-186

2 Aydin S, Abuzayed B, Yildirim H, Bozkus H, Vural M. Discal cysts of the lumbar spine: report of five cases and review of the literature. Eur Spine J 2010;19(10):1621-1626

3 Kang H, Liu WC, Lee SH, Paeng SS. Midterm results of percutaneous CT-guided aspiration of symptomatic lumbar discal cysts. AJR Am J Roentgenol 2008;190(5):W310-W314 